Reprod. Nutr. Dévelop., 1988, 28 (6 B), 1615-1628.

\title{
Avortements d'origine immunologique
}

\author{
Marie-Françoise REZNIKOFF-ETIEVANT
}

C.H.P./I.N.T.S

6. rue Alexandre Cabanel, 75015 Paris.

\section{Summary. Immunology of abortions.}

The immunology of pregnancy involves a series of systemic and above all local events, at the feto-maternal interface. These immunological events may explain the paradoxical non-rejection and development of the allogeneic conceptus within of the maternal body. The immunodeviation of the maternal immune system towards fetal tolerance may be altered or insufficient leading to true abortive diseases.

The alteration of this tolerance may be the result of auto-immune abnormalities, particularly when autoantibodies are discovered in woman's serum. These antibodies are the antithromboplastin, antiphospholipid and antinuclear antibodies. They can be responsible for abortion even if the clinical symptoms of the lupus disease are absent. Abortive events occur at all stages of pregnancy. Table 1 shows that abortions with autoantibodies are more frequent when the accident occurs in the late stage of pregnancy. The therapy with corticoids and aspirin will be modulated in connection with the results.

The second immune etiology is an insufficient production of immunological events usually involved in normal pregnancies. The current means available to detect this incompetency are imperfect because only systemic factors may be explored when local events are the most involved. We found a good correlation, in these women with recurrent early abortions of unkown etiology, between the production of anti-husband lymphocytes (AAP) and the success of a subsequent pregnancy (fig. 1).

Women who suffer from recurrent spontaneous abortions of unknown etiology, without autoimmune abnormalities and without antipaternal antibodies (AAP), may profit from a therapy using the husband's leucocyte injections, which allows them to give birth to a normal child in $85 \%$ of the cases, whereas without treatment the success rate is only $37 \%$ of the pregnancies (fig. 2). When this therapy is applied in accurate conditions, its inocuousness seems well established.

Another kind of this immunomodulator therapy has been reported : it uses unrelated donor leucocytes. Its efficiency seems to be similar to that of the husband's leucocyte injections.

L'immunologie de la grossesse met en jeu de nombreux mécanismes pour expliquer le paradoxe de la maintenance du fœtus au sein de l'organisme maternel. La femme enceinte n'est pas immunodéprimée comme cela avait été suggéré par Medawar en 1954. Bien au contraire, il semble tout à fait nécessaire qu'elle reconnaisse son fœtus comme étranger afin d'élaborer les éléments qui contribuent à le protéger des mécanismes de rejet.

Cette immunodéviation, du système immunitaire de la femme vers la tolérance active pour son fœtus, est le résultat de l'intervention d'une cascade de facteurs non spécifiques ou spécifiques des antigènes paternels du fœtus dont l'action est prédominante localement dans la région péri-placentaire. 
Dans certaines circonstances, une anomalie ou une déficience de cet équilibre harmonieux de l'immunotolérance peut être responsable d'une véritable maladie abortive d'origine immunologique. Pendant longtemps les couples affectés sont restés sans espoir de traitements appropriés; depuis quelques années, de nouvelles thérapeutiques peuvent dans certains cas leur être proposées.

On distingue actuellement deux groupes principaux d'avortements immunologiques:

1) Les avortements par anomalie autoimmune, impliquant l'organisme maternel, il existe un dysfonctionnement de son système immunitaire.

2) Les avortements par insuffisance des mécanismes de tolérance, impliquant le couple, exemple les femmes qui ont des enfants normalement avec un conjoint et des avortements répétés avec un autre conjoint.

\section{Immunité et grossesse.}

La tolérance immunologique de la mère pour le fœtus reste un phénomène encore imparfaitement connu. Les événements qui l'accompagnent sont multifactoriels, les premiers mis en évidence sont d'ordre systémique, mais il semble actuellement que les phénomènes locaux soient plus directement impliqués. II existe en effet des relations étroites et multiples entre les cellules de la muqueuse déciduale utérine et le sang maternel d'une part et les trophoblastes qui entourent et protègent le fœtus d'autre part.

\section{Evénements systémiques.}

1) Une première explication serait la production d'anticorps « facilitants » ou "bloquants » de natures diverses. De tels anticorps ont été observés chez la femme au cours de la grossesse normale et non, pour certains d'entre eux, chez les femmes faisant des fausses couches spontanées (FCS) répétées de causes méconnues (Suciu-Foca et al., 1983 ; Power et al., 1983 ; Rocklin et al., 1982 ; Mclntyre et Faulk, 1982). Cependant, ces anticorps ne sont pas des facteurs indispensables à l'évolution normale de la grossesse, témoin en est des grossesses menées à terme chez les femmes agammaglobulinémiques. Mais la production de tels anticorps pourrait toutefois être utile. Mowbray et al. (1987) et Reznikoff-Etievant et al. (1987a, b) ont pu observer que la réponse humorale contre les antigènes paternels du fœtus est corrélée avec le succès d'une nouvelle grossesse et celui de l'immunothérapie anti-abortive.

2) D'autres éléments sériques non spécifiques produits au cours de la grossesse normale contribuent vraisemblablement au maintien de la greffe fœto-placentaire par leur effet inhibiteur de certaines fonctions de rejet. Ces facteurs sont nombreux, citons : les hormones et notamment la progestérone et I'hCG et d'autres protéines associées à la grossesse qui possèdent in vitro une action inhibitrice de la transformation lymphoblastique ou de la cytotoxicité cellulaire (Fizet et Bousquet, 1983). 
3) L'immunité cellulaire est également déviée vers une immunité de tolérance. Chaouat et Voisin (1979) ont démontré, chez l'animal au cours de la gestation, la présence de cellules suppressives dont l'induction dépend d'un facteur placentaire. Chez la femme de telles cellules ont pu être mises en évidence par Engelman et al. (1978).

\section{Evénements locaux.}

Deux éléments sont au centre des phénomènes immunologiques de la tolérance de la mère pour son fœtus, ce sont le placenta et son trophoblaste d'une part et la muqueuse déciduale d'autre part.

1) Parmi les nombreuses fonctions du trophoblaste, voyons celles qui interfèrent dans les mécanismes immunitaires: a) II présente une résistance intrinsèque particulière à la cytotoxicité cellulaire. $b$ ) Chez la femme cette première propriété est renforcée par l'absence des antigènes d'histocompatibilité majeurs de classes I et II sur le syncytio et le cytotrophoblaste, de sorte que les éventuelles cellules cytotoxiques spécifiques de la mère ne les reconnaîtraient pas comme cible. c) Chez la souris, Head et Zuckerman (communication personnelle), ont pu démontrer in vivo la résistance des cellules placentaires aux anticorps cytotoxiques en présence de complément, les anticorps cytotoxiques seraient transformés en anticorps bloquants par inactivation de la partie de la molécule qui fixe le complément. $d$ ) Le cytotrophoblaste présente des antigènes monomorphes de classe 1, Ellis et al. (1986) et le syncytiotrophoblaste porterait les alloantigènes TLX (McIntyre et Faulk, 1982). Les uns ou les autres pourraient jouer un rôle particulier dans la stimulation d'éléments suppresseurs ou d'inhibiteurs locaux.

2) L'endomètre recrute en $2^{\mathrm{e}}$ phase du cycle des cellules T suppressives sous l'effet des hormones et au moment de l'implantation, sous l'effet du trophoblaste, d'autres cellules suppressives de plus petite taille (Clark et al., 1984). Les cellules $T$ helper (CD4+) inhibées dans leur fonction de production de I'IL2 (élément indispensable à la génération de cellules cytotoxiques) par un facteur appelé "facteur suppresseur afférent placentaire», seraient cependant capables sous l'influence d'antigènes du trophoblaste, de libérer des lymphokines possédant une action trophique pour les cellules placentaires, directement ou par l'intermédiaire des macrophages que l'on peut observer à son voisinage ainsi que le proposent Athanassakis et Wegman (1986).

Ainsi, certaines femmes ou plus précisément certains couples seraient prédisposés à présenter des anomalies de la reproduction telles que des avortements répétés si les antigènes placentaires provenant du père sont insuffisants à stimuler le système immunitaire de la femme. Cette conception est en accord avec l'augmentation d'homologie HLA observée chez les couples présentant des fausses couches inexpliquées, par rapport aux couples normalement fertiles (Reznikoff-Etievant et al., 1987b). Les antigènes HLA étudiés dans ces travaux ne sont pas directement à l'origine de la pathologie abortive; les antigènes directement impliqués (antigènes HLA monomorphes et/ou TLX) seraient génétiquement situés à leur proximité. 


\section{Fréquence des avortements immunologiques.}

La fréquence des avortements immunologiques n'est pas évaluée pour le moment, car cette entité est trop récente.

Dans la majorité des cas, le diagnostic d'avortement immunologique n'est actuellement évoqué qu'après avoir éliminé les causes classiquement connues : anomalies du corps utérin, incompétence cervicale, anomalies hormonales, infections locales, hypertension artérielle, diabète, anomalies chromosomiques et pathologie spermatique.

II convient de parler d'avortements à répétition après au moins trois fausses couches consécutives. II est clair que les risques de récidive augmentent avec le nombre d'accidents abortifs. Avec Malpas (1938), Eastman (1946) et Charvet et Mamelle (1985), on peut estimer très approximativement que $9 \%$ des premières grossesses sont interrompues spontanément, qu'après une première FCS le risque abortif est d'environ $22 \%$, puis de $38 \%$ après deux $\mathrm{FCS}$, de près de $70 \%$ après $3 \mathrm{FCS}$, et probablement de plus de $90 \%$ après $4 \mathrm{FCS}$.

Le bilan de l'étude de Stray-Pedersen (1984) montre sur un ensemble de 195 couples ayant présenté au moins trois avortements, que dans $56 \%$ des cas une des étiologies précédentes peut être retrouvée. II reste ainsi de l'ordre de $44 \%$ d'avortements spontanés répétés de cause inconnue. C'est donc dans ce groupe que les étiologies immunologiques sont actuellement étudiées.

\section{Avortements autoimmuns.}

Le retentissement du lupus érythémateux disséminé (LED) sur la vitalité du fœtus est un fait bien admis, comme l'atteste le pourcentage anormalement élevé d'avortements spontanés, de morts in utero et d'accouchements prématurés (environ 3 fois plus) au cours de cette affection. La fréquence d'une association LED et grossesse est faible. Morin et Gernigou (1970) l'estiment à 1 cas sur 1660 grossesses, Friedman et Rutherford (1956) à 1 cas sur 2000 grossesses. L'accident survient surtout lors de poussées évolutives de la maladie, le pronostic est meilleur lorsque la grossesse survient après une période de rémission d'au moins 6 mois. L'existence d'une néphropathie lupique est considérée comme un facteur prédisposant, la fréquence des pertes fœtales pouvant survenir dans $55 \%$ des cas voire plus ; mais le mécanisme reste incomplètement élucidé. Récemment ont été incriminés les anticorps anti-phospholipides. Ceux-ci constituent une famille complexe d'anticorps dont l'anti-prothrombinase ou anticoagulant circulant de type lupique ( $A C C$ ), l'anticardiolipine et le facteur anti-nucléaire, auto-anticorps retrouvés dans le lupus.

Il est important de noter que des avortements ou autres accidents fœtaux, le plus souvent répétés, peuvent être observés avant l'apparition de la maladie lupique; c'est dire l'importance de rechercher ces auto-anticorps en cas d'avortements spontanés répétés inexpliqués. Depuis la description par Nilson et al. (1975) d'une association entre ACC et mort in utero, de nombreuses études répertoriées (Dantal et al., 1988) ont confirmé cette corrélation qu'elle soit 
associée ou non à un LED. La fréquence de $20 \%$ d'avortements chez la femme lupique sans $A C C$ passe à $60 \%$ en présence d'ACC. II en est de même lorsque le lupus est uniquement biologique.

Soulier et Boffa (1980) ont décrit un syndrome associant thrombose, présence d'ACC et avortements spontanés sans signe de lupus. On relève dans la littérature de nombreuses observations d'une association ACC et FCS sans autre signe. Les résultats des études prospectives de l'autoimmunité chez des femmes présentant des FCS inexpliquées varient considérablement d'un auteur à l'autre, faisant état de la présence de l'un ou plusieurs de ces anticorps dans 9 à $40 \%$ des cas (Cowchock et al., 1986 ; Unander et Lindhom, 1987 ; Edelman et al., 1987 ; Reznikoff-Etievant et al., 1988). Ces variations sont probablement dues à des différences au niveau du recrutement des patientes et à l'introduction dans certaines séries d'accidents plus ou moins tardifs. En effet, dans une étude de 200 femmes ayant présenté des fausses couches spontanées, nous avons observé une incidence significativement plus importante de ces auto-anticorps lorsque les accidents sont tardifs (tabl. 1).

TABLEAU 1

Autoanticorps et fausses couches spontanées.

\begin{tabular}{lccc}
\hline & (n= 140$)$ & $(\mathrm{n}=30)$ & (III \\
& $(\mathrm{n}=30)$ \\
\hline Facteur antinucléaire & $4,1 \%$ & 0 & $19 \%$ \\
Anti-cardiolipide & $2,5 \%$ & $15 \%$ & $21 \%$ \\
Anti-prothrombinase & 0 & $4 \%$ & $7 \%$ \\
\hline TOTAL 11\%: & $6 \%$ & $15 \%$ & $35 \%$ \\
\hline Autres: & $0,8 \%$ & 0 & 0 \\
Anti-mitochondrie & 0 & $9 \%$ & 0 \\
Anti-réticulum endoplas & $1,6 \%$ & $4,5 \%$ & $8 \%$ \\
Anti-muscle lisse & $8 \%$ & $28 \%$ & $38 \%$ \\
\hline TOTAL 16\%: & & &
\end{tabular}

Groupe $1 \geqslant 3$ avortements précoces et pas d'enfant vivant.

Groupe II $\geqslant 3$ avortements précoces avec enfant vivant.

Groupe III $\geqslant 3$ avortements tardifs avec/sans enfant vivant.

II est important de rechercher les ACC et les anticardiolipides pendant la grossesse car ceux-ci peuvent être absents entre les grossesses.

Le mécanisme d'action des auto-anticorps n'est pas encore bien élucidé. Les $\mathrm{ACC}$ contrairement à ce que leur nom pourrait laisser entendre, n'entraînent généralement pas de manifestations hémorragiques, mais au contraire des thromboses vasculaires et notamment placentaires et déciduales, s'accompagnant d'infarcissement. Ces anticorps se fixent sur les phospholipides de 
l'endothélium vasculaire empêchant la production de prostacycline et par là même favorisant l'action de son antagoniste le thromboxane, d'où une hyperadhésivité plaquettaire.

Au plan thérapeutique on peut être amené à traiter aussi bien les sujets lupiques que les femmes asymptomatiques porteuses d'anticorps anti-phospholipides et ayant eu des antécédents d'avortements. En effet les chances de mener une grossesse normale en présence d'ACC serait de $2 \%$.

Le traitement consiste en corticothérapie aux doses usuelles avant ou pendant la grossesse en cas de lupus. Lorsque les signes biologiques sont isolés, de faibles doses peuvent être préconisées selon les cas, associées ou non à des doses faibles d'aspirine comme cela a été suggéré par Lubbe et al. (1983). Le traitement s'il est institué pendant la grossesse doit être précoce. L'efficacité de cette thérapeutique est difficile à estimer de façon précise étant donné l'absence d'étude en double aveugle. II n'est pas impossible que cette corticothérapie puisse être efficace dans un certain nombre de cas idiopathiques, une étude en double aveugle sera mise en route à ce sujet.

\section{Mécanismes de tolérance insuffisants.}

Parmi les mécanismes immunologiques qui contribuent à la maintenance de la greffe fœto-placentaire, les plus étudiés chez la femme en cas de fausses couches répétées, sont les facteurs périphériques.

Plusieurs anticorps, ayant une action supposée facilitante, retrouvés dans le sérum des femmes au cours de la grossesse normale, n'ont pas été observés dans le sérum des femmes qui font des avortements spontanés répétés sans causes apparentes : a) les anticorps anti-idiotypes qui bloquent des récepteurs HLA-DR présents sur les immunoblastes maternels stimulés par les antigènes paternels et décrits par Suciu-Foca et al. (1983); b) les anticorps anti-lymphocytes B, non anti-HLA, étudiés par Power et al. (1983); c) les anticorps bloquants, de spécificité non HLA, de nature lgG, mis en évidence par Rocklin et al. (1982). Ces anticorps inhibent la production de MIF (migrator inhibitor factor). Ils sont absorbés par les lymphocytes paternels, leur spécificité n'est pas connue.

Ainsi l'immunité anti-paternelle pourrait-elle refléter un état de tolérance acquise, favorable à l'évolution normale d'une grossesse. A l'inverse les femmes qui ne parviennent pas à s'immuniser risqueraient de rejeter plus fréquemment leur fœtus. Reznikoff-Etievant et al. (1987a, b) ont étudié l'immunisation anti-paternelle de 200 femmes ayant eu au moins trois fausses couches spontanées et suivi l'évolution d'une grossesse ultérieure en fonction des résultats de l'immunisation anti-paternelle. Dans cette étude ont été éliminées 22 femmes possédant des stigmates biologiques d'auto-immunité.

La recherche d'une immunité anti-paternelle a été réalisée selon le test de lymphocytotoxicité à partir de sérum maternel en présence des lymphocytes $T$ et B du mari. La présence d'anticorps anti-lymphocytes paternels (AAP) semble corrélée, chez ces femmes avec antécédents de FCS, avec une évolution favorable de la grossesse ultérieure (fig. 1). Les AAP sont actifs essentiellement sur les 
lymphocytes $\mathrm{B}$ du mari et à $37^{\circ} \mathrm{C}$. Leur spécificité n'a pu être définie, peut-être du fait de leur faible intensité dans la majorité des cas. Chez ces femmes, après au moins 3 FCS, nous avons observé ces anticorps chez $28 \%$ d'entre elles.

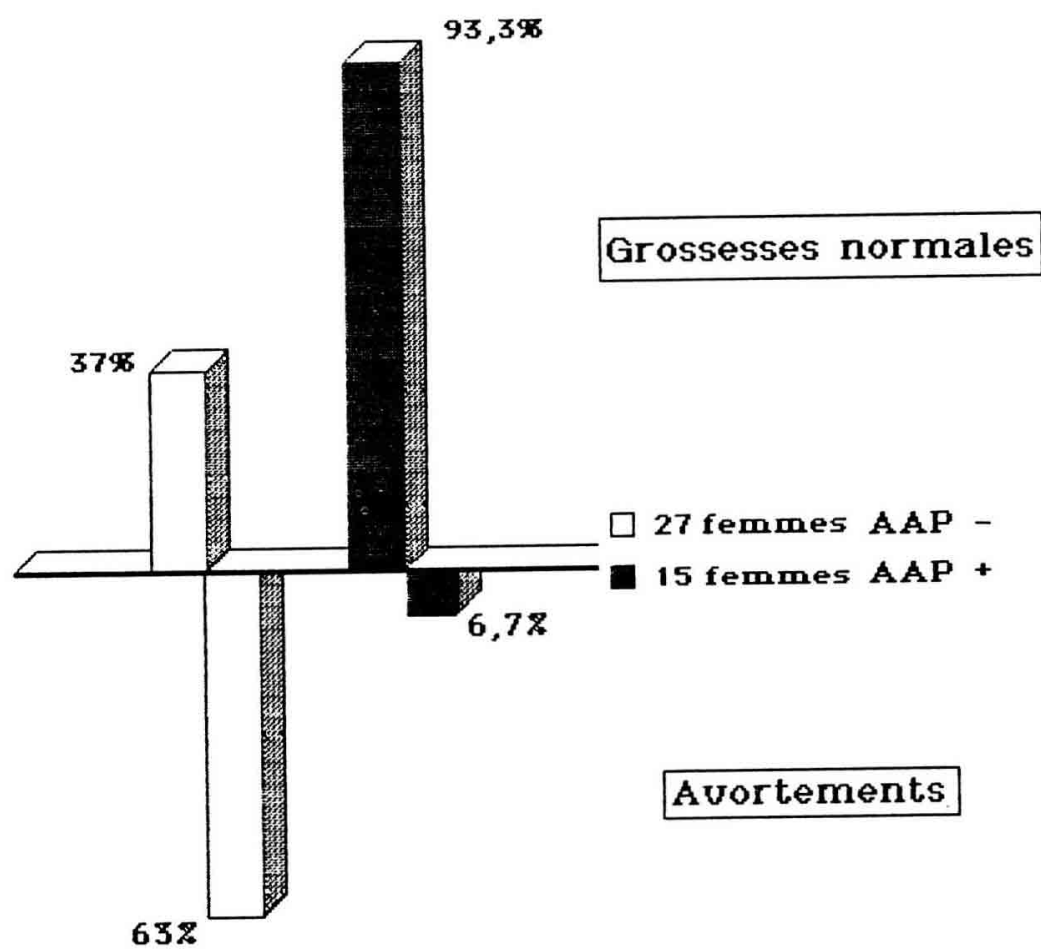

FIG. 1. - Evolution de 42 grossesses chez des patientes non traitées, en fonction de la présence ou non d'anticorps anti-paternels.

Bien que chez l'animal il ait été possible, comme nous l'avons déjà signalé, de démontrer que de tels anticorps pouvaient être transformés au contact des cellules throphoblastiques en anticorps bloquants, il n'est pas prouvé que ces anticorps participent à la tolérance foeto-placentaire. Quoi qu'il en soit, par leur présence, les AAP prouvent bien que la mère a reconnu un signal fœtal, étape importante pour le maintien et le développement normal de la grossesse.

Leur recherche permet cependant de sélectionner les femmes AAP négatives à traiter. Ces résultats sont en accord avec ceux de Mowbray et al. (1985).

\section{Traitement immuno-modulateur.}

Depuis 1981, un traitement transfusionnel peut être proposé dans de tels cas. Les résultats bénéfiques des transfusions avant la greffe d'organe sur la prise du greffon et la connaissance des antigènes portés à la fois par le trophoblaste et les

Reproduction, Nutrition, Développement, $n^{\circ} 6 \mathrm{~B} / 88-7$ 
lymphocytes dont le rôle serait primordial dans la régulation de la tolérance maternelle, ont incité à tenter une telle thérapeutique en cas d'avortements répétés idiopathiques.

Dans l'étude de Reznikoff-Etievant et al. (1975 et 1987a), 95 patients répondaient aux critères cliniques et biologiques déjà évoqués et ne possédaient pas d'AAP dans leur sérum. Ces femmes ont été immunisées avec les lymphocytes de leur mari, avant le début d'une nouvelle grossesse. Avant chaque injection un bilan de sécurité transfusionnel a été réalisé. Si la femme était CMV négatif et le mari CMV positif, le couple ne devait pas débuter une grossesse avant un délai de 8 semaines après la dernière injection et après un contrôle, afin d'éviter un risque de primo-infection en début de grossesse.

400 millions de lymphocytes du mari, préparés à partir d'un buffy-coat de plasmaphérèse, ont été injectés (environ 50 millions en SC et ID et le reste en IV). Une nouvelle recherche d'AAP a été effectuée 15 jours après chaque injection ; après séroconversion, les injections ont été interrompues. 3 injections ont été effectuées au maximum, même si la femme est restée AAP (-); actuellement, chez de telles patientes, une nouvelle injection est tentée en tout début de la nouvelle grossesse.

Parmi les grossesses qui ont débuté avant la fin $1987 \mathrm{chez}$ les femmes ainsi traitées, le taux global de grossesses menées à terme a été de $86 \%$. Le détail des résultats en fonction de l'apparition des AAP dans le sérum après les injections (fig. 2) montre que le taux de succès est encore plus élevé chez les femmes AAP + (92\%).

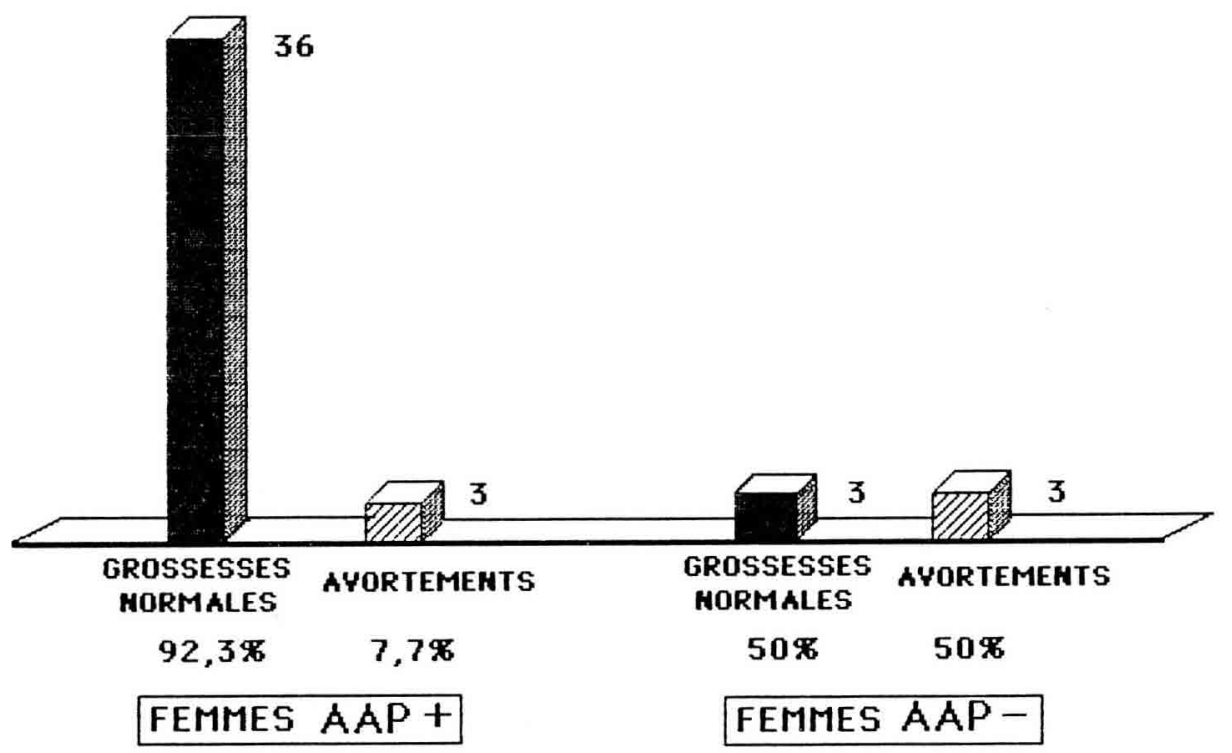

\section{GROSSESSES NORMALES}

FIG. 2. - Evolution des grossesses après injection des lymphocytes du mari. 
Les grossesses ont évolué normalement jusqu'à terme à l'exception d'une pour laquelle des signes de toxémie gravidique sont apparus vers la $32^{e}$ semaine ; un garçon de $2500 \mathrm{~g}$ est né par césarienne à 36 semaines de grossesse. Actuellement, cet enfant est en bonne santé à l'âge de 4 ans. Tous les autres enfants sont nés normalement entre 38 et 41 semaines avec un poids de naissance de $3255 \mathrm{~g} \pm 905 \mathrm{~g}$.

L'importance de l'immunisation antipaternelle (AAP) a été soulignée chez 6 patientes: 4 ont été enceintes avant la fin du traitement, celui-ci a été interrompu. Elles étaient AAP (-) et ont toutes les quatre refait une FCS. Le traitement a été alors poursuivi avec séroconversion $\operatorname{AAP}(+), 3$ d'entre elles ont été de nouveau enceintes et ont mené leur grossesse à terme. 2 étaient AAP ( - ) après 3 injections. Une nouvelle injection leur a été effectuée alors qu'elles étaient de nouveau enceintes. Elles sont devenues AAP $(+)$ en cours de grossesse et ont accouché normalement à terme.

Au cours de ces traitements, les chances de mener à terme une grossesse sont d'autant plus importantes que le délai qui la sépare de la dernière injection est plus court.

\section{Résultats obtenus par d'autres équipes.}

- Mowbray et al. $(1985,1987)$ sont les seuls à avoir mené une étude en double aveugle. Le protocole thérapeutique consiste à faire une seule injection d'environ 250 à 400 millions de lymphocytes du mari. Cette injection est effectuée à chaque patiente avant toute grossesse. Le protocole en double aveugle a été significatif lorsque $\mathbf{2 4}$ grossesses ont été enregistrées chez les patientes traitées avec leurs propres lymphocytes (groupe placebo), avec $37 \%$ de grossesses menées à terme et 22 femmes traitées avec les lymphocytes de leur mari, avec $77 \%$ de grossesses menées à terme. Etudiant l'effet de dose, ils ont pu déterminer qu'en dessous de 200 millions de lymphocytes injectés les risques d'échec étaient très importants. Lorsque l'injection a été réalisée aux alentours de la conception, le risque d'échec apparaît si elle est faite au-delà du $25^{\mathrm{e}}$ jour après la conception. Dans l'ensemble, le succès est de $75 \%$. Ces auteurs pensent que le fait de répéter les injections dans certains cas comme cela est pratiqué par la précédente équipe, serait probablement souhaitable. Près de 200 enfants sont nés après cette thérapeutique. L'état de santé des enfants a été comparé à celui d'enfants nés dans la même maternité et dans le même temps. Les résultats sont tout à fait comparables. Les poids de naissance ne montrent aucune particularité.

- Beer et al. (1981, 1987) ont été les instigateurs de ce traitement par injection des lymphocytes du mari. Leur protocole consistant à faire 2 injections en SC 40 millions de leucocytes à 6 semaines d'intervalle chez des femmes n'ayant pas développé de facteur bloquant. Le taux de succès est d'environ $79 \%$ et paraît également bien corrélé avec l'immunisation anti-paternelle d'anticorps bloquants. Chez une vingtaine de femmes n'ayant malgré tout pas développé d'anticorps bloquants après ce traitement, ces auteurs ont effectué une série d'injections de leucocytes d'un même donneur non apparenté au couple, 2 injections avant la grossesse et toutes les 6 semaines jusqu'à 24 semaines au 
cours de la grossesse. Sur 18 grossesses ainsi traitées, 8 ont évolué vers une nouvelle FCS et 10 femmes ont accouché. Lorsque la grossesse a évolué vers le terme, les facteurs bloquants étaient présents dans le sérum, tandis que chez les femmes qui font une nouvelle fausse couche, ce facteur n'a pas été retrouvé. Fait tout à fait particulier dans cette série, 5 enfants sont nés très hypotrophiques : 4 parmi les 112 enfants nés après traitement de la mère par lymphocytes du mari, l'un d'entre eux reste un cas très préoccupant et 1 parmi les 10 enfants nés des femmes traitées par la suite avec les lymphocytes d'un donneur non apparenté. Ce phénomène reste pour le moment inexpliqué. II est possible que le mode d'injection et la faible quantité de lymphocytes injectés, modalités particulières à ce protocole, puissent jouer un rôle. En effet, aucune autre équipe ne semble avoir eu ce type d'accident.

- Taylor et Faulk $(1981,1987)$ ont effectué des transfusions de leucocytes de différents donneurs. Le protocole consiste, sans sélection particulière de la femme, à transfuser le buffy-coat de 2 à 5 donneurs, deux avant fécondation puis toutes les 4 semaines jusque vers la $20^{\circ}$ semaine au cours de la grossesse. Cette méthode a l'inconvénient de faire appel à un grand nombre de donneurs différents pour une même patiente, multipliant de ce fait les risques transfusionnels ; de plus, la sélection des donneurs nécessite des examens relativement nombreux. Quoi qu'il en soit, ce traitement s'avère efficace dans $74 \%$ des cas.

- Unander et Lindhom (1986). Dans ce protocole, les femmes répondant aux critères cliniques et biologiques précédemment énoncés, ont reçu trois culots enrichis en leucocytes, à 2 mois d'intervalle. Une recherche d'anticorps bloquant la MLR (lymphocytes de la femme en présence de ceux du mari) réalisée avant et après le traitement a montré que les patientes qui possédaient des anticorps bloquants avant le traitement, risquaient presque toujours de faire une nouvelle FCS. Par contre, chez celles qui n'en possédaient pas, la grossesse après traitement avait près de $90 \%$ de chances d'évoluer jusqu'au terme. Il s'est avéré ultérieurement que dans le premier groupe de patientes, le titre des anticorps anti-cardiolipides était presque toujours élevé, attestant l'origine probablement autoimmune de ces fausses couches.

Le bénéfice apporté par l'immunothérapie dans la maladie abortive immunologique a pu être vérifié dans différents modèles animaux: chez la souris (fig. 3). Les avortements observés chez les souris femelles CBA croisées avec des souris DBA2 peuvent être jugulés si les souris CBA sont préalablement immunisées avec des lymphocytes de souris $B a l b / c$. Les antigènes d'histocompatibilité (HLA, chez l'homme) des souris DBA2 et Balb/c sont particuliers en ce sens qu'elles ont les mêmes antigènes majeurs, mais diffèrent par les antigènes mineurs (Kiger et al., 1985). Des résultats équivalents ont été obtenus chez les souris B10 croisées avec des mâles B10.A; mais dans ce cas le phénomène est dépendant des antigènes majeurs d'histocompatibilité, et la régression des avortements est obtenue par l'injection préalable de lymphocytes ou de sérum de souris B10 immunisées par B10.A (Chaouat, 1987). Enfin chez la jument, l'implantation d'embryons d'âne est normalement voué à l'échec. Si la jument est traitée avec des lymphocytes d'âne l'implantation peut alors aboutir (Allen et al., 1987). 


\section{MODELES MURINS}

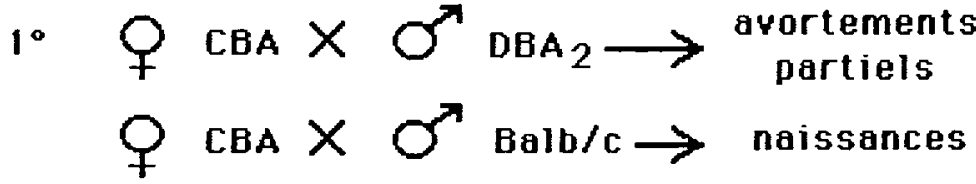

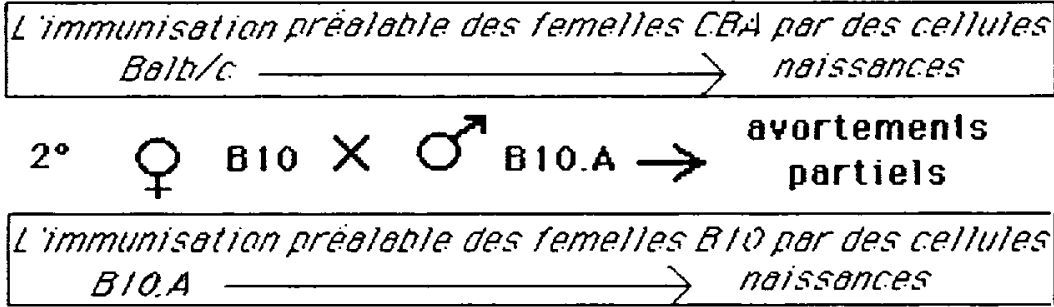

MODELE JUMENT/ANE

L'implantation d’embryons d’âne chez la jument

avortements

1 immumisation de la jument avec des cellules d'âne après
limplantatian $\stackrel{\text { naissances }}{\longrightarrow}$

FIG. 3. - Immunotherapie des avortements chez l'animal.

\section{Conclusion.}

II apparaît de plus en plus évident que des mécanismes immunologiques peuvent expliquer un certain nombre de fausses couches auparavant restées sans étiologie. Les unes peuvent être dues à des causes auto-immunes notamment lorsque des anticorps anti-phospholipides sont identifiés chez de telles femmes. Les autres sont dues à des déficiences des mécanismes de la tolérance fœto-maternelle. A chacune de ces étiologies correspondent des thérapeutiques appropriées.

II reste, dans tous ces cas, encore beaucoup à découvrir pour préciser aussi bien les mécanismes que les modalités du diagnostic et du traitement.

En outre, il est bien évident que certaines formes d'avortements spontanés sont d'étiologies intriquées, où l'on peut rencontrer simultanément des causes morphologiques, hormonales ou autres associées à des causes auto et alloimmunes voire même psychologiques. Il convient donc de rester ouvert à chacune de ces étiologies et l'on peut être amené, dans certaines circonstances, à associer de façon personnalisée, différentes thérapeutiques. 
Sur le plan scientifique, l'étude des fausses couches immunologiques présente un intérêt qui dépasse leur seul propos, elle peut en effet déboucher sur des domaines aussi variés que le cancer et la transplantation.

$27^{e}$ Réunion de la Société française pour l'Etude de la Fertilité. Paris, 29, 30 sept., $1^{\text {er }}$ oct. 1988.

\section{Références}

ALLEN W. R., KYDD J., DONALDSON W. L., ORIOL J. G., ANTCZAK D. F., 1987. Expression of maternal immune response to fetal antigens in equine pregnancy. In, Immunologie de la reproduction : relation materno-fotale. Coll. INSERM, 154, 255-270.

ATHANASSAKIS I., WEGMANN T. G., 1986. The immunotrophic interaction between maternal T cells and fetal trophoblast/macrophages during gestation. In Reproductive immunology, Elsevier Sci. Publ., pp. 91-98.

BEER A. E., QUEBBEMAN J. F., AYERS J. T., HAINES R. F., 1981. Major histocompatibility complex antigens, maternal immune response and chorionic habitual abortion in humans. Am. J. Obstet. Gynecol., 141, 987-999.

BEER A. E., SHEKAR S. S., QUEBBEMAN J. F., XIAOYU ZHU, 1987. Paternal and non paternal leucocyte immunization in women with recurrent spontaneous abortions : immune responses and subsequent pregnancy outcome. In, Immunologie de la reproduction : relation maternofotale. Coll. INSERM, 154, 161-178.

CHARVET F., MAMELLE N., 1985. Les fausses couches spontanées à répétition. Rev. fr. Gynecol. Obstet., 80, 555-558.

CLARK D. A., SLAPSYS R. M., CROY B. A., ROSSANT J., 1984. Immunoregulation of host versus graft responses in the uterus. Immunol. Today, 5, 11-13.

CHAOUAT G., VOISIN G. A., 1979. Regulatory T cell populations in pregnancy. I. Evidence for suppressive activity of the early phase of MLR. J. Immunol., 122, 1383-1388.

CHAOUAT G., 1987. 2 modèles d'avortements d'origine immunitaire chez la souris de laboratoire ; mécanismes abortifs, modalités et mécanismes de traitement par immunisation contre un mâle, relié ou non relié, suivant les différences antigéniques pères-mères. In Immunologie de la reproduction : relation materno-fotale. Coll. INSERM, 154, 243-254.

COWCHOCK S., SMITH J. B., GOCIAL B., 1986. Antibodies to phospholipids and antinuclear antigens in patients with repeated abortions. Am. J. Obstet. Gynecol., 155, 1002-1010.

DANTAL J., DANTAL P., SAJOT P., LOPES P. F., 1988. Anticoagulants circulants et grossesse : une association à risque. J. Gynecol. Obstet. Biol. Reprod., 17, 317-324.

EASTMAN N. J., 1946. Habitual abortion, p. 262. In J. V. MERGS, S. STURGIS, Progress in gynecology. Grune and Stratten, New York.

EDELMAN Ph., ROUQUeTtE A. M., SUREAU Cl., 1987. Auto-immunity and abortive disease. Dans, Immunologie de la reproduction: relation materno-fœtale. Coll. INSERM, 154. 225-232.

ELLIS S. A., SARGENT I. L., REDMAN C. W. G., MCMICHAEL A. J., 1986 . Evidence for a novel HLA antigen found on human extravillous trophoblast and a choriocarcinoma cell line. Immunology, 59, 595-601.

ENGELMAN E. G., McMICHAEL A. J., McDEVITT H. O., 1978. Suppression of the mixed lymphocyte reaction in man by soluble $T$ cell factor: specificity of the factor for both the responder and the stimulator. J. exp. Med., 147, 1037-1045.

FIZET D., BOUSQUET, 1983. Absence of a factor blocking cellular cytotoxicity in the serum of women with recurrent abortion. Br. J. Obstet. Gynaecol., 90, 453-456.

FRIEDMAN E. A., RUTHERFORD J. W., 1956. Pregnancy and lupus erythematosus. Obstet. Gynecol., 8, 601-610. 
KIGER N., CHAOUAT G., KOLB J. P., WEGMANN T. G., GUENNET J. L., 1985. Immunogenetic studies of spontaneous abortion in mice. I. Preimmunisation of the mother with allogeneic spleen cells. J. Immunol., 134, 2966-2972.

LUBBE W., BUTLER W., PALMER S., LIGGINS G., 1983. Fetal survival after prednisone suppression of maternal lupus anticoagulant. Lancet, i, 1361-1363.

MALPAS P., 1938. A study of abortion sequences. J. Obstet. Gyn. Br. Emp., 45, 932-941.

McINTYRE J. A., FAULK W. P., 1982. Allotypic trophoblast-lymphocyte cross-reactive (TLX) cell surface antigens. Human Immunol., 4, 27-35.

MORIN P., GERNIGOU G., 1970. Lupus érythémateux et grossesse. Rev. Prat., 18, $2965-2978$.

MOWBRAY J. F., LIDDELL H., UNDERWOOD J. L., GIBBINS C., REGINALD P. W., BEARD R. W., 1985. Controlled trial of treatment of recurrent spontaneous abortion by immunization with paternal cells. Lancet, i, 941-943.

MOWBRAY J. F, UNDERWOOD J. L., MICHEL M., FORBES P. W., BEARD R. W., 1987. Immunisation with paternal lymphocytes in women with recurrent miscarriage. Lancet, ii, 679-680.

NILSON J. M., ASTEDT B., HEDNER U., BERESIN D., 1975. Intra-uterine death and circulating anticoagulant (antithromboplastin). Acta. med. scand., 197, 153-159.

POWER D. A., MASON R. J., STEWART K. N., NOBLE N. D., Mac LEOD A., SHEWAN G., GATTO G. R. D., 1983. B lymphocyte antibodies associated with successful pregnancy: evidence for MHC linkage. Transplant. Proc., 15, 890-892.

REZNIKOFF-ETIEVANT $M$. F., SIMMONEY N., JANAUD A., DARBOIS Y., NETTER A., 1985. Treatment of spontaneous abortions by immunisation with paternal leucocytes. Lancet, i, 1398.

REZNIKOFF-ETIEVANT M. F., DURIEUXI., HUCHET J., SALMON Ch., NETTER A., 1987a. Paternal leucocyte injections in recurrent spontaneous abortion. Lancet, ii, 1960.

REZNIKOFF-ETIEVANT M. F., DURIEUXI., HUCHET J., SALMON Ch., NETTER A., 1987b. Recurrent spontaneous abortions, HLA antigen sharing and anti-paternal immunity, Immunotherapic assay. In, Immunologie de la reproduction: relation materno-fotale. Coll. INSERM, 154, $187-202$.

REZNIKOFF-ETIEVANT M. F., JARRAYA M. A., FERRER LE CEUR F., ALBENGRES E., ABUAF N., 1988. Autoimmune investigations in recurrent spontaneous miscarriages. Am. J. Reprod. Immunol. Microb., 16, 87.

ROCLIN R. E., KITZMILLER J. L., GARAVOY M. R., 1982. Materno-fetal relation. II. Further characterization of an immunologic blocking factor that develops during pregnancy. Clin. Immunol. Immunopath. 22, 303-315.

SOULIER J. P., BOFFA M. C., 1980. Avortements à répétition, thromboses et anti-coagulant circulant anti-thromboplastine. Nouv. Presse méd., 9, 859-864.

STRAY-PEDERSEN B., STRAY-PEDERSEN S., 1984. Etiologic factors and subsequent reproductive performance in 195 couples with a prior history of habitual abortion. Am. J. Obstet. Gynecol., 148, 140-146.

SUCIU-FOCA N., REED E., ROHOWYSKY C., KUNG P., KING D. W., 1983. Anti-idiotypic antibodies to HLA receptors induced by pregnancy. Proc. nat. Acad. Sci. USA, 80, 230-234.

TAYLOR C., FAULK W. P., 1981. Prevention of recurrent abortion with leucocyte transfusions. Lancet, ii, 68-69.

TAYLOR C. G., FAULK W. P., Mac INTYRE J. A., HILL J. G., Mac LACHLAN N., 1987. The TLX hypothesis and treatment of spontaneous recurrent abortion by immunization with 3rd party lymphocytes. In, Immunologie de la reproduction : relation materno-fatale. Coll. INSERM, 154, 203-212.

UNANDER A. M., LINDHOM A., 1986. Transfusions of leucocyte-rich erythrocyte concentrates: A successful treatment in selected cases of habitual abortion. Am. J. Obstet. Gynecol., 154, 516-520.

UNANDER A. M., NORBERG R., HALM L., ARFORS L., 1987. Anticardiolipin antibodies and complement in ninety-nine women with habitual abortion. Am. J. Obstet. Gynecol., 156, 114-119. 